\title{
Brisk walking offsets the increase in postprandial TAG concentrations found when changing to a diet with increased carbohydrate
}

\author{
Stephen F. Burns*, Adrianne E. Hardman and David J. Stensel \\ School of Sport and Exercise Sciences, Loughborough University, Loughborough, Leicestershire LE11 $3 T U$, UK \\ (Received 29 April 2008 - Revised 9 October 2008 - Accepted 15 October 2008 - First published online 25 November 2008)
}

We tested the hypothesis that 30 min of brisk walking daily would offset the increase in plasma TAG concentrations associated with substituting dietary fat for carbohydrate. Fourteen subjects (six males) aged 57 (SD 5) years underwent three $4 \mathrm{~d}$ trials in a counterbalanced order: (i) $4 \mathrm{~d}$ on a typical UK diet (40\% energy from fat, $45 \%$ carbohydrate and $15 \%$ protein); (ii) $4 \mathrm{~d}$ on an isoenergetic diet but substituting fat for carbohydrate in line with the present recommendations (30\% fat, $55 \%$ carbohydrate and $15 \%$ protein); (iii) $4 \mathrm{~d}$ on the same recommended diet with 30 min of brisk walking each day. The food provided for the first $3 \mathrm{~d}$ of each trial was isoenergetic with each volunteer's previously determined daily energy intake. On day 4, the subjects consumed breakfast, lunch and an early evening meal, equivalent in total to $90 \%$ of their daily energy intake. Blood samples were collected and substrate utilisation and energy expenditure were determined in the fasted state and for $9 \mathrm{~h}$ postprandially. Substrate utilisation differed significantly among trials $(P=0.003)$; RER was higher on the recommended diet trial than during either of the other two trials $(P=0.012$ and 0.021 for the UK diet and recommended diet with walking, respectively). The rise in plasma TAG concentrations over the day was steeper on the recommended diet trial than on the other two trials (trial $\times$ time interaction, $P=0 \cdot 040$ ). The increase in postprandial TAG concentrations associated with substituting dietary fat for carbohydrate was offset by 30 min of brisk walking daily.

TAG: Walking: Carbohydrates: Postprandial lipaemia

Replacing dietary fat with carbohydrate is recommended in the prevention of CHD because of the potential to reduce plasma total and LDL-cholesterol ${ }^{(1)}$. However, high-carbohydrate diets frequently increase fasting ${ }^{(2-6)}$ and postprandial $^{(7-10)}$ plasma TAG concentrations, which are risk factors for $\mathrm{CHD}^{(11-15)}$. The postprandial period may be of particular importance because most individuals on a Western diet spend the majority of their day in a non-fasting state. The postprandial period may promote atherosclerosis through: (1) an accumulation of plasma TAG-rich lipoprotein remnants; (2) catabolism of HDL and (3) formation of small, dense $\mathrm{LDL}^{(16)}$.

Given that high-carbohydrate diets reduce plasma cholesterol, it is important to investigate ways to offset increases in plasma TAG concentrations seen when changing to these diets. One potential strategy is exercise. Endurance-trained athletes exhibit low fasting TAG concentrations and high HDL concentrations despite consuming diets high in carbohydrate $^{(17)}$. Moreover, in overweight men who followed a low-fat high-carbohydrate hypoenergetic diet over 1 year, those individuals who included regular walking or jogging (mean distance $15 \mathrm{~km} /$ week) demonstrated significantly greater decreases in fasting TAG and significantly greater increases in HDL-cholesterol than men who followed the diet only ${ }^{(18)}$. In overweight women following the same programme, only those following a diet plus exercise regimen showed significant decreases in plasma TAG and significant increases in HDL-cholesterol compared with sedentary controls who did not change their diet ${ }^{(18)}$.

Previous investigations demonstrate that exercise can reduce the postprandial TAG response to a high-fat meal when following a short-term low-fat high-carbohydrate diet ${ }^{(19,20)}$. In these studies, subjects consumed a high-fat meal after $3 \mathrm{~d}$ on either a low- or high-carbohydrate diet. Postprandial plasma TAG concentrations were higher after the high- than low-carbohydrate diets. Inclusion of daily exercise, however, abolished the increase in TAG concentrations with the high-carbohydrate diets. While these studies demonstrate that exercise can reduce TAG concentrations after a high-fat meal with a preceding high-carbohydrate diet, they do not show whether exercise reduces TAG concentrations after consuming meals with increased carbohydrate content. This is an important issue for individuals who wish to lower their cholesterol concentration while preventing an increase in their TAG concentration. It is also important as the alternative to replacing dietary fat with carbohydrate is replacing saturated fat with unsaturated fat, a recommendation distrusted by some investigators $^{(21)}$ and one which could send a confusing message to the public.

The present study, therefore, tested the hypothesis that $30 \mathrm{~min}$ of brisk walking daily could offset the increase in

Abbreviation: AUC, area under the plasma concentration $v$. time curve.

* Corresponding author: Stephen F. Burns, fax: +1 412692 8531, email stephen.burns@chp.edu 
plasma TAG concentrations associated with a $10 \%$ change in dietary energy (carbohydrate for fat). The dietary change and activity dose were chosen as they are achievable for most adults making lifestyle changes and because they reflect present public-health policies ${ }^{(1,22-25)}$. To test these changes, we employed a short-term diet and exercise intervention in middle-aged adults. Lipoproteins are accentuated with short-term dietary changes as the body responds to changes in diet composition ${ }^{(10)}$. Middle-aged adults were used because of the large increase in CHD morbidity and mortality that occurs in this age group compared with younger individuals $^{(26)}$.

\section{Subjects and methods}

\section{Subjects}

The study was approved by Loughborough University's Ethical Advisory Committee and all volunteers gave written informed consent after an explanation of study procedures. A total of twenty-two volunteers were recruited over 2 years from the local community by advertisements and written letters to individuals who had previously taken part in experiments in our laboratory and who indicated their willingness to be informed about future studies. Eight subjects withdrew from the study. One was subsequently found to be a smoker and hence did not fulfil the selection criteria. Five subjects withdrew due to personal or work commitments and two subjects were withdrawn because of technical difficulties during data collection. This left six male and eight female participants aged 45-63 years who completed the study. Inclusion criteria were: (i) aged 45-65 years; (ii) no personal history of CVD, metabolic disease or dyslipidaemia and (iii) resting arterial blood pressure $<140 / 90 \mathrm{~mm} \mathrm{Hg}$; (iv) no extreme dietary habits (proportion of energy from dietary fat $>25 \%$ or $<45 \%$, as assessed by a preliminary 3-d weighed food intake inventory of 2 weekdays and 1 weekend day); (v) non-vegetarian; (vi) non-smoker (vii) not taking drugs known to influence lipid or carbohydrate metabolism; (viii) recreationally active but not engaging in strenuous exercise and (ix) (women only) more than 2 years without a menstrual period. Some physical characteristics of the subjects are provided in Table 1. Two female subjects were taking hormone replacement therapy but made no changes during their participation in the study.

\section{Study design}

A schematic of the study design is provided in Fig. 1. Each subject spent $4 \mathrm{~d}$ following one of three interventions in a counterbalanced order separated by at least 2 weeks. These interventions comprised: (i) $4 \mathrm{~d}$ on a typical UK diet $(\mathrm{UK})^{(27)}$; (ii) $4 \mathrm{~d}$ on an isoenergetic diet lower in fat and higher in carbohydrate in line with the present recommendations (recommended) ${ }^{(1,24,25)}$; (iii) $4 \mathrm{~d}$ on the same recommended diet with the addition of one $30 \mathrm{~min}$ session of brisk walking each day (recommended + walking). The food provided for the first $3 \mathrm{~d}$ of each trial was isoenergetic to each volunteer's daily habitual energy intake. On the fourth day, the subjects attended the laboratory and $90 \%$ of the energy intake on the other $3 \mathrm{~d}$ was provided. The day before their first dietary intervention, the subjects were asked to record the food they ate at home and to repeat this food intake the day before beginning the next two interventions. The subjects refrained from alcohol consumption and any exercise other than that prescribed during the intervention period. There was a 14-d washout period between interventions where subjects resumed their usual physical activity and dietary habits.

\section{Preliminary testing}

Exercise. One preliminary exercise test was conducted to establish the steady-state relationship between the submaximal oxygen uptake $\left(\dot{\mathrm{VO}}_{2}\right)$ and the treadmill gradient during uphill walking at a constant speed. The maximum oxygen uptake $\left(\dot{\mathrm{VO}}_{2 \max }\right)$ was estimated by the extrapolation of the $\dot{\mathrm{VO}}_{2} /$ heart rate relationship obtained during the test to the agepredicted maximum heart rate $(220$ - age in years).

Dietary analysis. In order to provide diets isoenergetic to each volunteer's daily habitual energy intake, all participants completed a 3-d weighed food intake inventory of their habitual diet on 2 weekdays and 1 weekend day before beginning the main trials. Food inventories were analysed for energy and nutrients using a computerised version (Comp-Eat 5.0; Nutrition Systems, London, UK) of food composition tables $^{(28)}$. Analysis of these food inventories showed that the subjects' normal diet provided 9.2 (SD 3.4) $\mathrm{MJ} / \mathrm{d}$, with 27.4 (SD 6.8) \% of energy as fat (9.0 (SD 3.8) \% as saturated fat), 54.2 (SD 6.6) \% as carbohydrate (23.2 (SD 7.8) \% as simple sugars and 30.7 (SD 9.4) \% as complex carbohydrate) and

Table 1. Physical characteristics of the subjects

(Mean values and standard deviations)

\begin{tabular}{|c|c|c|c|c|c|c|}
\hline & \multicolumn{2}{|c|}{ Females $(n 8)$} & \multicolumn{2}{|c|}{ Males ( $n 6)$} & \multicolumn{2}{|c|}{ All subjects $(n 14)$} \\
\hline & Mean & SD & Mean & SD & Mean & SD \\
\hline Age (years) & $57 \cdot 6$ & $4 \cdot 0$ & $56 \cdot 8$ & $6 \cdot 4$ & $57 \cdot 3$ & $5 \cdot 0$ \\
\hline Height $(m)$ & 1.61 & 0.07 & 1.82 & 0.08 & 1.70 & 0.13 \\
\hline Body mass $(\mathrm{kg})$ & $65 \cdot 8$ & $7 \cdot 8$ & $90 \cdot 1$ & $28 \cdot 8$ & $76 \cdot 2$ & 22.5 \\
\hline BMI $\left(\mathrm{kg} / \mathrm{m}^{2}\right)$ & $25 \cdot 3$ & $2 \cdot 4$ & $26 \cdot 8$ & 5.5 & $26 \cdot 0$ & 4.0 \\
\hline Systolic blood pressure $(\mathrm{mmHg})$ & 125 & 9 & 124 & 7 & 124 & 8 \\
\hline Diastolic blood pressure $(\mathrm{mmHg})$ & 81 & 6 & 84 & 6 & 83 & 6 \\
\hline Waist circumference (m) & 0.82 & 0.07 & 0.95 & 0.21 & 0.88 & 0.16 \\
\hline Predicted $\dot{\mathrm{VO}}_{2} \max (\mathrm{ml} / \mathrm{kg} /$ per min) & $28 \cdot 3$ & $6 \cdot 1$ & $39 \cdot 1$ & $6 \cdot 8$ & $33 \cdot 3$ & $8 \cdot 3$ \\
\hline
\end{tabular}




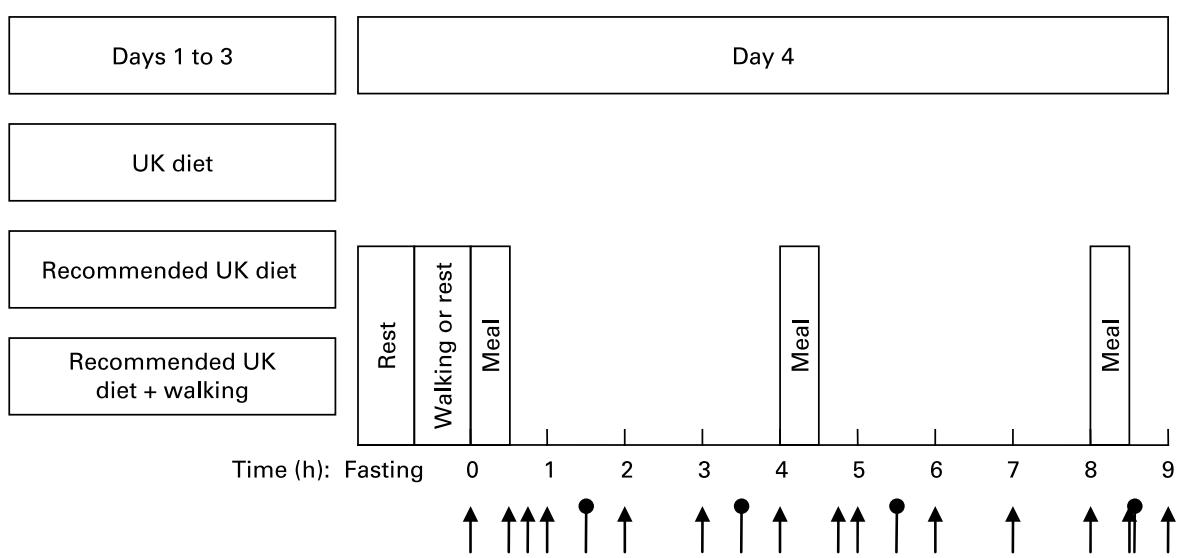

Fig. 1. A schematic of the protocol. UK diet: $40 \%$ fat; $45 \%$ carbohydrate; $15 \%$ protein. Recommended UK diet: $30 \%$ fat; $55 \%$ carbohydrate; $15 \%$ protein. Walking: $30 \mathrm{~min}$ of 'brisk' walking daily. Blood sample ( $\mathbf{\uparrow}$ ) and expired air collection ( $\boldsymbol{\varphi}$ ).

16.7 (SD 2.8) $\%$ as protein. The mean quantity of macronutrients consumed each day was as follows: fat 69 (SD 33) g/d; carbohydrate 311 (SD 115) g/d; protein 90 (SD 29) g/d.

\section{Experimental diets}

The composition of the experimental diets is provided in Table 2. All food items were provided for the participants, together with food weighing scales (EKS International SAS, Wittisheim, France) and detailed instructions about methods of preparation and cooking. The participants prepared three meals and one or two snacks each day, to a detailed menu, weighing each item. The importance of following the diets 'to the gram' was explained to the subjects. Researchers were in regular contact with the volunteers to ensure compliance. On the fourth day of each intervention, during laboratory studies, food was prepared by the researchers. As the day spent in the laboratory was of shorter duration than the other $3 \mathrm{~d}$ of the intervention, only $90 \%$ of the energy intake of the other $3 \mathrm{~d}$ was provided to avoid overfeeding participants. Meals in the laboratory were divided into breakfast (20\% energy), lunch (50\% energy) and an early evening meal (30\% energy). The UK diet aimed to provide $40 \%$ energy as fat, $45 \%$ as carbohydrate and $15 \%$ as protein ${ }^{(27)}$. The recommended diet aimed to provide energy as $30 \%$ fat,

Table 2. Composition of the experimental diets

(Mean values and standard deviations)

\begin{tabular}{|c|c|c|c|c|}
\hline \multirow{2}{*}{ Component } & \multicolumn{2}{|c|}{ UK diet } & \multicolumn{2}{|c|}{ Recommended die } \\
\hline & Mean & SD & Mean & SD \\
\hline Energy $(\mathrm{MJ} / \mathrm{d})$ & $9 \cdot 2$ & 3.4 & 9.2 & 3.4 \\
\hline Carbohydrate (\%) & \multicolumn{2}{|c|}{46.9} & \multicolumn{2}{|c|}{$56 \cdot 7$} \\
\hline Simple (\%) & \multicolumn{2}{|c|}{$16 \cdot 7$} & \multicolumn{2}{|c|}{$26 \cdot 8$} \\
\hline Complex (\%) & \multicolumn{2}{|c|}{30.2} & \multicolumn{2}{|c|}{29.9} \\
\hline Fat (\%) & \multicolumn{2}{|c|}{$39 \cdot 3$} & \multicolumn{2}{|c|}{$30 \cdot 3$} \\
\hline Saturated fat (\%) & \multicolumn{2}{|c|}{$9 \cdot 4$} & \multicolumn{2}{|c|}{$7 \cdot 7$} \\
\hline Protein (\%) & \multicolumn{2}{|c|}{$13 \cdot 7$} & \multicolumn{2}{|c|}{$13 \cdot 0$} \\
\hline Carbohydrate (g) & 257.7 & $95 \cdot 1$ & 311.5 & $115 \cdot 0$ \\
\hline Fat $(\mathrm{g})$ & $96 \cdot 0$ & 35.4 & 74.0 & $27 \cdot 3$ \\
\hline Protein $(\mathrm{g})$ & $75 \cdot 3$ & $27 \cdot 8$ & 71.4 & $26 \cdot 4$ \\
\hline Cholesterol (mg) & 168 & 62 & 147 & 54 \\
\hline
\end{tabular}

$55 \%$ carbohydrate and $15 \%$ protein $^{(1,24,25)}$, with $<10 \%$ of energy from saturated fat. There were slight differences in the composition of the diet we gave the subjects and the afore-mentioned proportions (Table 2). The sources of carbohydrate in the diets were cereals, bread, orange juice, sugar, boiled sweets, biscuits, fruits (apples, pears, bananas and tinned pineapple), rice, potatoes and crisps. The change in carbohydrate between the UK and recommended diets was mostly achieved by manipulating the amounts of these foods rather than by introducing different foods. The cholesterol content and ratio of saturated, monounsaturated and polyunsaturated fat was held constant between the diets.

\section{Exercise sessions}

For the recommended + walking intervention, the participants came to the laboratory on each of the $4 \mathrm{~d}$ they were on the diet and walked on a motorised treadmill for $30 \mathrm{~min}$. The subjects were asked to walk at a self-selected 'brisk' pace, which was both comfortable and sustainable for $30 \mathrm{~min}$. Heart rate was monitored using short-range telemetry (SPORT-TESTER; Polar Electro, Kempele, Finland) and rating of perceived exertion was recorded ${ }^{(29)}$. Expired air samples were collected every 15 min into 200 litre Douglas bags (Plysu Protection Systems, Milton Keynes, UK). Whole-body carbohydrate and fat utilisation rates and energy expenditure in exercise were calculated from indirect calorimetry, without measurement of urinary nitrogen excretion ${ }^{(30)}$. On days $1-3$ of the intervention, walking was not taken at a specified time during the day. We did not specify the time of walking on these days as the present experimental protocol aimed to mimic a short period of lifestyle change in individuals. On day 4 of the intervention, walking was performed in the morning, before the consumption of breakfast and after fasting blood samples were taken, and resting energy expenditure was measured (see 'Postprandial protocol'). Walking was performed on the morning of day 4 as there is some uncertainty as to whether exercising during the postprandial period is effective at reducing lipaemia ${ }^{(31,32)}$. In addition, our participants were to consume three meals within $8 \mathrm{~h}$ and we wished to avoid gastrointestinal discomfort during the postprandial period. Only activities of daily living were permitted during the two non-exercise interventions. 


\section{Postprandial protocol}

The participants arrived at the laboratory by car after a $12 \mathrm{~h}$ fast, at approximately 08.00 hours. A cannula was introduced into a forearm or antecubital vein and the subject rested quietly in a supine position for $10 \mathrm{~min}$, after which a baseline blood sample was obtained. The subject then lay quietly while RMR and RER were measured using a ventilated hood attached to an automated metabolic cart. The participants then consumed their breakfast. The first bite of breakfast was taken as time 0 . Four hours later, the participants were provided with lunch and $4 \mathrm{~h}$ after this an early evening meal. Further blood samples were collected at hourly intervals until $9 \mathrm{~h}$ after the start of breakfast, with additional smaller samples at 30 and $45 \mathrm{~min}$ after breakfast, $45 \mathrm{~min}$ after lunch and $30 \mathrm{~min}$ after the early evening meal. The cannula was kept patent by flushing with non-heparinised saline $(9 \mathrm{~g}$ $\mathrm{NaCl} / \mathrm{l})$. Further measurements of RMR and RER were obtained at $1.5,3.5,5.5$ and $8.5 \mathrm{~h}$ after the start of breakfast using the ventilated hood. The subjects rested or worked quietly during the observation period and consumed only water between meals.

\section{Energy expenditure at rest and during the postprandial period}

RMR and RER were measured using a ventilated hood attached to an automated metabolic cart (GEM Europa Scientific, NutrEn Technology Ltd, Manchester, UK). The subject was comfortably positioned on the bed and RMR was measured. The subject spent $15 \mathrm{~min}$ under the hood, with the first $5 \mathrm{~min}$ acting as a habituation and calibration period and the next $10 \mathrm{~min}$ for data collection. The ventilated hood was calibrated for each measurement of RMR and RER. Mean volumes of $\mathrm{O}_{2}$ and $\mathrm{CO}_{2}$ were used to calculate energy expenditure during the measurement period ${ }^{(33)}$. The RER was used to assess the fraction of energy expenditure contributed by carbohydrate and fat, assuming that variations in RER were not affected by protein oxidation.

\section{Analytical methods}

Blood samples were collected into pre-cooled 9 and $4.5 \mathrm{ml}$ potassium EDTA monovettes (Sarstedt, Leicester, UK) and kept on ice until centrifugation within 15 min. Plasma was stored at $-80^{\circ} \mathrm{C}$ until analysed at a later date for plasma total and HDL-cholesterol (fasted samples only), glucose, 3hydroxybutyrate (Randox Laboratories Ltd, Crumlin, UK) and NEFA (Wako Chemicals GmbH, Neuss, Germany) by enzymatic colorimetric methods with the use of an automated centrifugal analyser (Cobas Mira Plus; Roche Diagnostic Systems, Welwyn Garden City, Hertfordshire, UK). Using the same analyser, the concentrations of TAG in plasma were measured enzymatically, with correction for free glycerol ${ }^{(34)}$. Plasma insulin concentration was determined using a solidphase ${ }^{125}$ I RIA available in a commercial kit (ICN Pharmaceuticals, Inc., Costa Mesa, CA, USA). Radioactivity was measured using an automated gamma counting system (Cobra II; Packard Instrument Company Inc., Downers Grove, IL, USA). All samples from all trials for each subject were always analysed in the same batch. The within-batch CV was $0.7 \%$ for total cholesterol, $1.8 \%$ for HDL-cholesterol,
$0.9 \%$ for glucose, $2.7 \%$ for 3-hydroxybutyrate, $0.8 \%$ for NEFA, $1.8 \%$ for TAG and $5.7 \%$ for insulin.

\section{Calculations and statistics}

Results were analysed using statistical software (SPSS 10.0, SPSS Inc., Chicago, IL, USA). Plasma concentrations in the fasted state were compared among trials using a one-way ANOVA for repeated measures. Differences in postprandial concentrations of measured plasma constituents, energy expenditure and RER over time were compared among trials using two-way ANOVA (trial $\times$ time) for repeated measures. In addition, summary measures of the postprandial responses were calculated as the total areas under the plasma concentration $v$. time curves (AUC) using the trapezoidal rule. Incremental AUC was calculated for plasma TAG responses as the total AUC minus the fasting value extrapolated over $9 \mathrm{~h}$. Summary measures were compared among interventions using one-way ANOVA for repeated measures. Multiple $t$ tests with Bonferroni adjustment (significance values multiplied by the number of $t$ tests comparisons performed) were used to determine where significant differences identified by oneway ANOVA occurred. A 5\% level of significance was adopted throughout. Data are expressed as means and standard deviations unless stated.

\section{Results}

\section{Responses to treadmill walking}

Mean speed during treadmill walking over $4 \mathrm{~d}$ on the exercise trial was 6.4 (SD 0.4) km/h (4.0 mph). The $\mathrm{VO}_{2}$ during walking was 17.6 (SD 2.1) $\mathrm{ml} / \mathrm{kg} /$ per min, which represented $53 \%$ of the predicted $\dot{\mathrm{VO}}_{2}$ max. Heart rate averaged 114 (SD 15) beats/min and mean ratings of perceived exertion were 11 (SD 1). Gross energy expenditure for each exercise session was 0.87 (SD 0.29) MJ (207 kcal), with $36 \%$ of the energy derived from fat and $64 \%$ from carbohydrate.

\section{Plasma concentrations in the fasted state}

No difference was observed among interventions in concentrations of measured fasting plasma constituents on day 4 of each trial (Table 3).

\section{Postprandial responses}

Changes over the postprandial period. Plasma TAG concentrations over day 4 of each trial are shown in Fig. 2. There was no effect of trial for plasma TAG concentrations. This was possibly because the observed power to detect a trial effect for TAG from the data within the present study was only 0.377. Plasma TAG concentrations differed over time (main effect of time, $P<0 \cdot 001)$. The rise in plasma TAG concentrations over the day was steeper on the recommended trial than on the other two trials, particularly, towards the end of the day (trial $\times$ time interaction, $P=0 \cdot 040$ ). The observed power to detect this interaction was $0 \cdot 943$. Post hoc analysis revealed six time points at which differences between the $\mathrm{UK}$ and recommended $(1,2,3$ and $8 \mathrm{~h})$ or recommended + walking and recommended trials (1 and $9 \mathrm{~h}$ ) occurred. 
Table 3. Concentrations of measured plasma constituents in the fasted state for subjects after $3 \mathrm{~d}$ on a typical UK diet (UK diet), a recommended diet (recommended) or the recommended diet plus $30 \mathrm{~min}$ of daily walking (recommended + walking)

(Mean values and standard deviations, $n$ 14)

\begin{tabular}{|c|c|c|c|c|c|c|c|}
\hline & \multicolumn{2}{|c|}{ UK diet } & \multicolumn{2}{|c|}{ Recommended } & \multicolumn{2}{|c|}{$\begin{array}{l}\text { Recommended }+ \\
\text { walking }\end{array}$} & \multirow[b]{2}{*}{$P$} \\
\hline & Mean & SD & Mean & SD & Mean & SD & \\
\hline TAG $(\mathrm{mmol} / \mathrm{l})$ & 0.97 & 0.58 & $1 \cdot 12$ & 0.69 & 1.01 & 0.73 & 0.197 \\
\hline Total cholesterol $(\mathrm{mmol} / \mathrm{l})$ & $6 \cdot 87$ & 1.69 & $6 \cdot 65$ & $1 \cdot 80$ & $6 \cdot 53$ & $1 \cdot 73$ & 0.337 \\
\hline HDL-cholesterol (mmol/l) & 1.43 & 0.41 & 1.36 & 0.36 & 1.39 & 0.41 & 0.356 \\
\hline Insulin (pmol/l) & 144 & 79 & 134 & 69 & 138 & 82 & 0.515 \\
\hline Glucose (mmol/l) & $5 \cdot 83$ & 0.84 & 5.92 & 0.94 & 6.09 & 0.70 & 0.450 \\
\hline NEFA (mmol/l) & 0.55 & $0 \cdot 16$ & 0.58 & 0.22 & 0.63 & $0 \cdot 19$ & 0.342 \\
\hline 3-Hydroxybutyrate $(\mathrm{mmol} / \mathrm{l})$ & 0.13 & 0.07 & 0.11 & 0.07 & $0 \cdot 16$ & $0 \cdot 11$ & 0.121 \\
\hline
\end{tabular}

After adjustment for multiple comparisons using the Bonferroni method, the only difference to remain significant was between the recommended and exercise trials at $9 \mathrm{~h}$ $(P<0.001$; Fig. 2).

Plasma insulin, glucose, NEFA and 3-hydroxybutyrate responses over day 4 of the trial are shown in the Appendix. Although not statistically significant, plasma glucose tended to be higher on the recommended + walking trial between breakfast and lunch (main effect of trial, $P=0 \cdot 052$ ). No difference was observed among trials in insulin concentrations. Glucose (main effect of time, $P<0.001$ ) and insulin (main effect of time, $P<0.001$ ) concentrations changed throughout the day on all trials peaking shortly after each meal. Concentrations of NEFA or 3-hydroxybutyrate did not differ among trials. Both NEFA (main effect of time, $P<0.001$ ) and 3 -hydroxybutyrate (main effect of time, $P<0.001$ ) followed the same pattern of response over the day, rising to a peak before meals. The fall in NEFA concentrations immediately after breakfast was less steep and the rise was greater before lunch on the recommended + walking trial than the dietonly trials (trial $\times$ time interaction, $P=0 \cdot 004$ ).

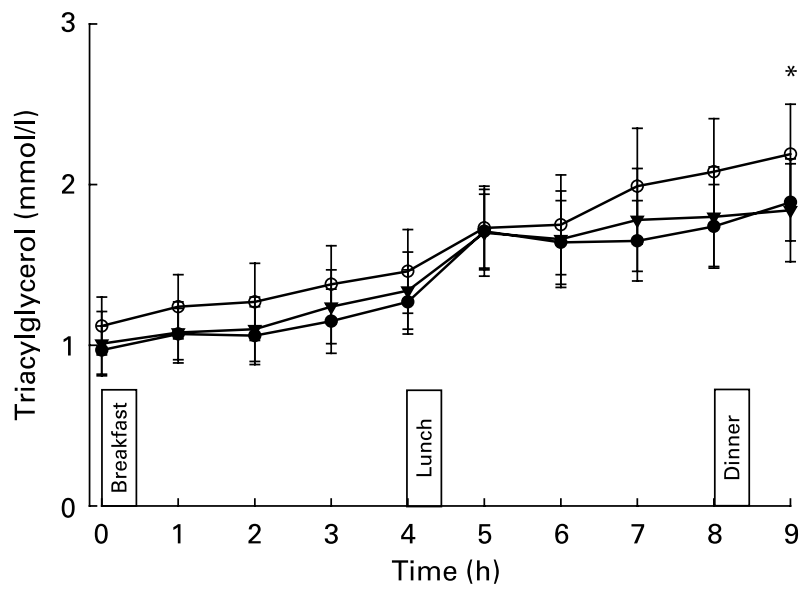

Fig. 2. Plasma TAG concentrations for $9 \mathrm{~h}$ while consuming (i) a typical UK diet $(\bullet)$, (ii) the recommended diet $(O)$ or (iii) the recommended diet with 30 min of brisk walking $(\mathbf{\nabla})$. Values are means with their standard errors ( $n$ 14). Main effect of time $(P<0.001)$; trial $\times$ time interaction $(P=0.040)$. ${ }^{*} \mathrm{~A}$ significant difference was found between the recommended and recommended + walking trial $(P<0.001)$.
Summary data. Nine-hour AUC for measured plasma constituents on day 4 of each diet is provided in Table 4. No difference was observed in the total or incremental AUC for TAG. Glucose AUC was significantly different among trials. Post hoc analysis revealed that this difference occurred between the recommended and the recommended + walking trials, with values tending to be higher on the recommended + walking trial $(P=0 \cdot 018)$. No significant difference was observed in the AUC among trials for any other measured plasma constituents.

\section{Energy expenditure and substrate utilisation}

There were no differences in RMR among trials at baseline or over the postprandial period on day 4. Energy expenditure changed over the day rising in the morning after breakfast and dropping before lunch before rising again throughout the afternoon on all trials (main effect of time, $P<0.001$ ).

Baseline substrate utilisation did not differ among trials but differed significantly over the day (main effect of trial, $P=0.003$; Fig. 3). Post hoc tests revealed that the RER was higher on the recommended than the UK $(P=0 \cdot 012)$ or recommended + walking trial $(P=0 \cdot 021)$, indicating a greater proportion of energy metabolised from carbohydrate on the recommended diet. The RER followed a similar pattern of response over the day to the RMR on all trials (main effect of time, $P<0 \cdot 001)$.

\section{Discussion}

The present study demonstrated that changing the source of $10 \%$ of energy in a typical UK diet from fat to carbohydrate, in line with the present recommendations, led to a subtle rise in postprandial TAG concentrations, particularly towards the end of the trial days. Importantly, we demonstrated that $30 \mathrm{~min}$ of brisk walking each day offsets the small elevation in TAG concentrations exhibited in middle-aged, normally active volunteers. This finding is practical for most individuals making lifestyle changes, as brisk walking is specified as one means of achieving the recommended level of physical activity ${ }^{(22,23)}$.

Fasting TAG concentrations have long been known to be related to the proportion of energy consumed from dietary carbohydrate in cross-sectional studies of different populations ${ }^{(4,5)}$ or in populations where low-fat high-carbohydrate 
Table 4. Nine-hour area under the plasma concentration $v$. time curve for measured plasma constituents in subjects after $3 d$ on a typical UK diet (UK diet), a recommended diet (recommended) or the recommended diet plus 30 min of daily walking (recommended + walking)

(Mean values and standard deviations, $n$ 14)

\begin{tabular}{|c|c|c|c|c|c|c|c|}
\hline & \multicolumn{2}{|c|}{ UK diet } & \multicolumn{2}{|c|}{ Recommended } & \multicolumn{2}{|c|}{$\begin{array}{l}\text { Recommended }+ \\
\text { walking }\end{array}$} & \multirow[b]{2}{*}{$P$} \\
\hline & Mean & SD & Mean & SD & Mean & SD & \\
\hline TAG $(\mathrm{mmol} \times 9 \mathrm{~h} / \mathrm{l})$ & $12 \cdot 71$ & $7 \cdot 02$ & $14 \cdot 57$ & 9.05 & $13 \cdot 13$ & 8.52 & 0.170 \\
\hline Incremental TAG $(\mathrm{mmol} \times 9 \mathrm{~h} / \mathrm{l})$ & 4.03 & $2 \cdot 70$ & 4.55 & $3 \cdot 10$ & 4.07 & $2 \cdot 41$ & 0.466 \\
\hline Insulin (pmol × 9h/l) & 5232 & 2718 & 4988 & 2324 & 4801 & 1883 & 0.192 \\
\hline Glucose $(\mathrm{mmol} \times 9 \mathrm{~h} / \mathrm{l})$ & $66 \cdot 29$ & $10 \cdot 51$ & 64.89 & 9.79 & $70.02^{*}$ & 7.44 & 0.026 \\
\hline NEFA $(\mathrm{mmol} \times 9 \mathrm{~h} / \mathrm{l})$ & 1.99 & 0.61 & 1.72 & 0.81 & 2.08 & 0.65 & 0.099 \\
\hline 3-Hydroxybutyrate $(\mathrm{mmol} \times 9 \mathrm{~h} / \mathrm{l})$ & 0.54 & 0.23 & 0.47 & 0.23 & 0.62 & 0.25 & $0 \cdot 116$ \\
\hline
\end{tabular}

${ }^{\star}$ Recommended + walking $v$. recommended $(P=0.018)$.

diets are introduced ${ }^{(2,3)}$. Even though mean fasting TAG concentrations were higher on the recommended than the UK or recommended + walking trial, no statistical significance was seen among trials in the present study. Thus, the modest exchange of fat for carbohydrate that we used to represent realistic lifestyle changes in the present study did not increase fasting TAG concentrations. This observation has previously been observed in at least one study where small increases in carbohydrate in the diet did not change fasting lipoprotein concentrations ${ }^{(35)}$. In studies with large dietary increases in carbohydrate, however, fasting TAG concentrations have increased ${ }^{(10,19,20)}$. One possibility for the present finding is that $3 \mathrm{~d}$ of dietary intervention was not long enough to produce significant changes in fasting TAG. However, previous studies ${ }^{(10,19,20)}$ with dietary interventions of the same time duration but greater proportions of carbohydrate $(70 \%)$ have found significant changes in fasting TAG, suggesting that it is the proportion of carbohydrate and not the length

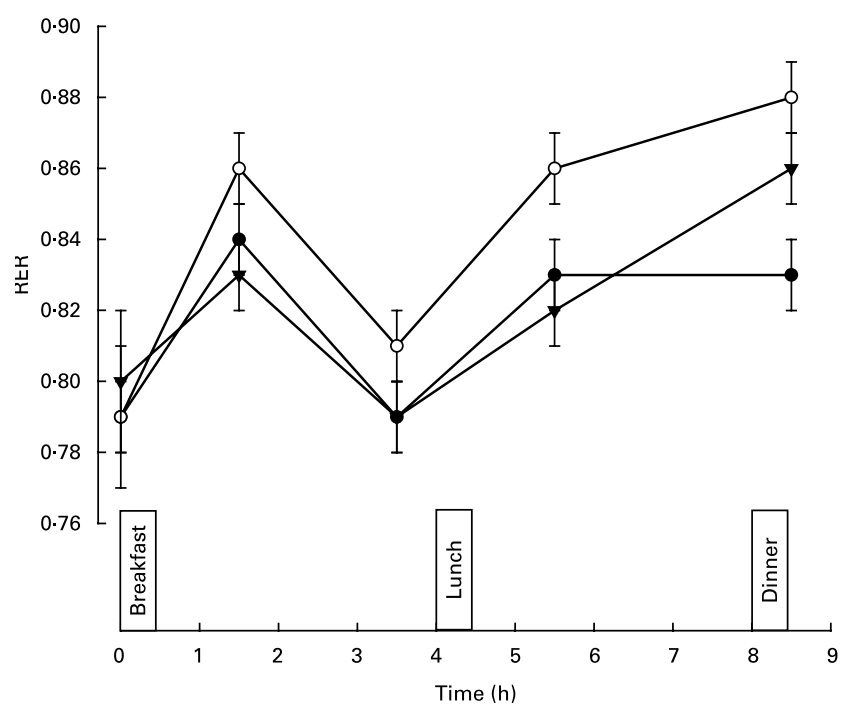

Fig. 3. Respiratory exchange ratio for $9 \mathrm{~h}$ while consuming (i) a typical UK diet $(\bullet)$, (ii) the recommended diet $(\bigcirc)$ or (iii) the recommended diet with $\mathbf{3 0}$ min of brisk walking $(\boldsymbol{\nabla})$. Values are means with their standard errors ( $n$ 14). Main effect of trial $(P=0.003)$; main effect of time $(P<0.001)$. Recommended diet $v$. typical UK diet $(P=0.012)$; recommended diet $v$. recommended diet + walking $(P=0.021)$. of the dietary intervention that may have been the deciding factor in the present study. An alternative explanation may be related to our subjects' background diet, which was on average already very similar to the recommended diet as evidenced by their 3-d weighed food inventory. These individuals may have been conscious of their lifestyle or diet, and so the small changes we made when providing the recommended diet may have had little impact on their fasting TAG concentrations. Certainly, there is some evidence that changes in fasting TAG concentrations with diet may be transient ${ }^{(36)}$.

Postprandial TAG concentrations have also been shown to increase in both healthy subjects ${ }^{(9,10,19,20)}$ and subjects with non-insulin-dependent diabetes mellitus ${ }^{(7,8)}$ when the proportion of carbohydrate intake in the diet has been increased from a period of $3 \mathrm{~d}$ to 6 weeks. A number of these studies $^{(9,10,19,20)}$ in healthy subjects gave $60-70 \%$ carbohydrate in the diet - a proportion greater than that used in the present study and much greater than that seen in a typical Western diet. Such a large change in the proportion of macronutrient intake is unlikely to be attainable for most individuals and may exaggerate the degree of postprandial lipaemia in comparison with more realistic dietary changes. Thus, we chose to feed our subjects a smaller proportion of energy from carbohydrate to reflect lifestyle changes that are in line with the present recommendations and attainable by most individuals. These changes produced a very small but significant rise in postprandial TAG concentrations in the latter stages of the experimental days. Thus, despite the potential cholesterol-lowering benefits of substituting carbohydrate for fat, TAG concentrations are still increased in the short term. Whether this undesirable effect is maintained for more than a transient period of initial dietary change ${ }^{(36)}$, or whether gradually phasing in carbohydrate for fat in small increases ${ }^{(35)}$ can prevent rises in postprandial TAG concentrations, is uncertain. As noted in the previous paragraph, however, it is possible that we did not observe greater changes in TAG concentrations between the diets we used because our subjects' background diet was similar to the recommended diet.

Previous studies have demonstrated that exercise can offset increases in TAG after consuming high-fat meals preceded by $3 \mathrm{~d}$ of short-term high-carbohydrate feeding in healthy young men $^{(19)}$ and postmenopausal women ${ }^{(20)}$. The energy expenditure of the exercise in those studies (1.58 and 1.46 MJ, respectively) was considerably greater than that used in the present study 
because of the differences in either exercise intensity or duration. In addition, the proportion of carbohydrate fed in those studies $(70 \%)$ was considerably greater than that we used. The present study adds to these previous investigations by demonstrating that a more attainable exercise volume ${ }^{(22,23)}$ can offset increases in postprandial TAG concentrations with more realistic increases in dietary carbohydrate. In addition, it demonstrates that exercise can offset these increases in response to carbohydrate meals per se rather than a high-fat meal fed after consuming a short-term high-carbohydrate diet.

Exercise can ameliorate increases in fasting TAG from high-carbohydrate diets ${ }^{(20,37,38)}$. Although fasting TAG concentrations were lowered $(0.11 \mathrm{mmol} / \mathrm{l}$ reduction) when walking was performed in conjunction with the recommended diet in the present study, this reduction was not significant. Nonetheless, it is still possible that exercise reduced the contribution of endogenous hepatic TAG during the postprandial period. High-carbohydrate diets probably accelerate hepatic TAG secretion by suppressing NEFA oxidation in the $\operatorname{liver}^{(39)}$. The decreased RER across the postprandial period on the recommended + walking intervention compared with the recommended trial suggests that fat oxidation was increased, probably in response to the energy deficit created by the exercise. Although RER reflects whole-body fat oxidation, it does not seem unreasonable to suggest that fat oxidation was also increased in the liver. Certainly, when exercise has been added to a short-term high-carbohydrate diet previously $^{(20)}$, decreased concentrations of postprandial hepatic TAG have been observed after the subjects consumed a highfat meal. One piece of evidence against this hypothesis in the present study is that plasma 3-hydroxybutyrate concentrations, a marker of fat oxidation in the liver, were not increased with the addition of walking to the recommended diet.

Plasma NEFA concentrations fell more slowly after breakfast on the recommended + walking trial than that on the other trials. Possibly adipose tissue TAG breakdown was slower to fall post-exercise because of increased skeletal muscle or liver energy requirements. The steeper rise in NEFA concentrations before lunch could also be to account for the energy deficit in these tissues. Interestingly, NEFA concentrations in the afternoon did not differ among trials. It may be that as exogenous TAG delivery to the muscle was at its greatest later in the day, the muscle was less reliant on endogenous energy from adipose tissue TAG breakdown. Plasma TAG concentrations were certainly at their highest in the afternoon reflecting the slower entry of exogenous TAG into the system. Moreover, skeletal muscle lipoprotein lipase, the capillary-bound enzyme responsible for hydrolysing TAG for uptake into the tissue, has a delayed increase after exercise and the activity of this enzyme is not thought to be at its greatest for at least $8 \mathrm{~h}$ after exercise, which may explain the higher reliance on endogenous fat sources during the morning ${ }^{(40)}$.

Previous studies have demonstrated that postprandial reductions in TAG are determined by the energy expenditure of the previous exercise ${ }^{(41-43)}$. It is possible that if our subjects had been left to eat under conditions of free energy intake, they may have replenished some or all the energy deficit created by the walking when it was added to the recommended diet, negating the reduction in lipaemia. Nonetheless, exercise is known to reduce postprandial lipaemia over and above that produced by an energy deficit alone ${ }^{(44)}$.
In addition, endurance-trained athletes exhibit low plasma fasting $^{(17)}$ and postprandial ${ }^{(45)}$ TAG concentrations despite consuming diets high in carbohydrate.

Conversely, two recent investigations, in normal-weight white women ${ }^{(46)}$ and overweight white men ${ }^{(47)}$, which have replaced the energy deficit created by an exercise bout with a post-exercise meal, have found that postprandial lipaemia was not attenuated in response to a high-fat meal the morning after. Interestingly, this was not the case in black women where exercise was still effective at reducing TAG concentrations despite replacement of the energy deficit ${ }^{(46)}$. Thus, there is some debate as to whether exercise can reduce postprandial lipaemia without a concomitant energy deficit. Possibly the effect is mediated by race, age, training status or the energy expenditure of the prior exercise.

As with fasting TAG, it is possible that changes to diet and exercise in the present study were not of sufficient size or duration to cause significant differences in fasting total or HDL-cholesterol. Nonetheless, the change in mean concentrations of total and HDL-cholesterol observed among the trials was as expected with these interventions. In comparison with the UK trial, the mean total cholesterol was reduced on the recommended diet and further reduced on the recommended + walking intervention, while HDL-cholesterol concentration was reduced on the recommended diet, but the addition of walking increased its concentration nearer to that observed on the UK diet. Over a longer diet and intervention period, such changes could well translate into positive health benefits for an individual.

In conclusion, the present study demonstrates that $30 \mathrm{~min}$ of brisk walking daily is sufficient to offset the small rise in TAG associated with changing from a typical UK diet to one that is lower in fat and higher in carbohydrate as frequently recommended. This finding may be important for individuals who wish to receive the cholesterol-lowering benefits of lower-fat diets while at the same time preventing any increases in TAG. It is also important that the changes to diet and exercise described are achievable by most individuals in the population.

\section{Acknowledgements}

The present work was supported by a British Heart Foundation Project grant no. PG/2000120. The authors would like to thank all the subjects for their voluntary participation in the present research. We also gratefully acknowledge the help of Professor Peter Jones, Dr Laura Barrett, Mr Adam Hassani and Ms Caroline Pearce with data collection and Ms Lisa Pierce for designing, analysing and prescribing the diets in the present study. D. J. S. and A. E. H. were responsible for the study design. All authors contributed to the data collection. S. F. B. and D. J. S. were responsible for the data analysis. All authors were involved in the preparation and review of the manuscript and accept full responsibility for its contents. None of the authors report any conflict of interest with respect to the present work.

\section{References}

1. Krauss RM, Eckel RH, Howard BV, et al. (2000) AHA dietary guidelines. Revision 2000: a statement for healthcare professionals from the nutrition committee of the American Heart Association. Circulation 102, 2284-2299. 
2. Farquhar JW, Frank A, Gross RC, et al. (1966) Glucose, insulin, and triglyceride responses to high and low carbohydrate diets in man. J Clin Invest 45, 1648-1656.

3. Ginsberg H, Olefsky JM, Kimmerling G, et al. (1976) Induction of hypertriglyceridemia by a low-fat diet. J Clin Endocrinol Metab 42, 729-735.

4. Connor WE, Cerqueira MT, Connor RW, et al. (1978) The plasma lipids, lipoproteins, and diet of the Tarahumara Indians of Mexico. Am J Clin Nutr 31, 1131-1142.

5. West CE, Sullivan DR, Katan MB, et al. (1990) Boys from populations with high-carbohydrate intake have higher fasting triglyceride levels than boys from populations with high-fat intake. Am J Epidemiol 131, 271-282.

6. Mensink RP \& Katan MB (1992) Effect of dietary fatty acids on serum lipids and lipoproteins: a meta-analysis 27 trials. Arterioscler Thromb 12, 911-919.

7. Blades B \& Garg A (1995) Mechanisms of increase in plasma triacylglycerol concentrations as a result of high carbohydrate intakes in patients with non-insulin-dependent diabetes mellitus. Am J Clin Nutr 62, 996-1002.

8. Chen YDI, Coulston AM, Zhou M-Y, et al. (1995) Why do high-carbohydrate diets accentuate postprandial lipemia in patients with NIDDM? Diabetes Care 18, 10-16.

9. Jeppesen J, Schaaf P, Jones C, et al. (1997) Effect of low-fat, high-carbohydrate diets on risk factors for ischemic heart disease in postmenopausal women. Am J Clin Nutr $\mathbf{6 5}$, 1027-1033.

10. Koutsari C, Malkova D \& Hardman AE (2000) Postprandial lipemia after short-term variation in dietary fat and carbohydrate. Metabolism 49, 1150-1155.

11. Hokansen JE \& Austin MA (1996) Plasma triglyceride level is a risk factor for cardiovascular disease independent of high-density lipoprotein cholesterol level: a meta-analysis of population-based prospective studies. J Cardiovasc Risk 3, 213-219.

12. Sarwar N, Danesh J, Eiriksdottir G, et al. (2007) Triglycerides and the risk of coronary heart disease: 10,158 incident cases among 262,525 participants in 29 Western prospective studies. Circulation 115, 450-458.

13. Patsch JR, Miesenböck G, Hopferwieser T, et al. (1992) Relation of triglcyeride metabolism and coronary artery disease. Studies in the postprandial state. Arterioscler Thromb 12, 1336-1345.

14. Bansal S, Buring JE, Rifai N, et al. (2007) Fasting compared with nonfasting triglycerides and risk of cardiovascular events in women. J Am Med Assoc 298, 309-316.

15. Nordestgaard BG, Benn M, Schnohr P, et al. (2007) Nonfasting triglycerides and risk of myocardial infarction, ischemic heart disease, and death in men and women. J Am Med Assoc 298, 299-308.

16. Karpe F \& Hamsten A (1995) Postprandial lipoprotein metabolism and atherosclerosis. Curr Opin Lipidol 6, 123-129.

17. Williams C (1998) Dietary macro- and micronutrient requirements of endurance athletes. Proc Nutr Soc 57, 1-8.

18. Wood PD, Stefanick ML, Williams PT, et al. (1991) The effects on plasma lipoproteins of a prudent weight-reducing diet with or without exercise, in overweight men and women. New Engl $J$ Med 325, 461-466.

19. Koutsari C \& Hardman AE (2001) Exercise prevents the augmentation of postprandial lipaemia attributable to a low-fat high-carbohydrate diet. Br J Nutr 86, 197-205.

20. Koutsari C, Karpe F, Humphreys SM, et al. (2001) Exercise prevents the accumulation of triglyceride-rich lipoproteins and their remnants seen when changing to a high-carbohydrate diet. Arterioscl Thromb Vasc Biol 21, 1520-1525.

21. Connor WE \& Connor SL (1997) Should a low-fat, high-carbohydrate diet be recommended for everyone? The case for a lowfat, high-carbohydrate diet. New Engl J Med 337, 562-563.
22. Department of HealthPhysical Activity, Health Improvement and Prevention (2004) A Report from the Chief Medical Officer. Crown Copyright.

23. Haskell WL, Lee I-M, Pate RR, et al. (2007) Physical activity and public health, updated recommendations for adults from the Amercian College of Sports Medicine and the Amercian Heart Association. Circulation 116, 1081-1093.

24. Committee on Medical Aspects of Food Policy, Department of Health (1991) Dietary Reference Values for Food Energy and Nutrients for the United Kingdom. Report on Health and Social Subjects no. 37. London: H.M. Stationery Office.

25. US Department of Health and Human Services/US Department of Agriculture (2005), www.healthier.gov/dietaryguidelines (accessed 17 January 2008).

26. British Heart Foundation Statistics Website. Deaths by cause, sex and age, 2005, United Kingdom. www.heartstats.org (accessed 17 January 2008).

27. Department for Environment Food and Rural Affairs (2007) UK Purchases and Expenditure on Food and Drink and derived Energy and Nutrient Intakes in 2005-06. http://statistics.defra. gov.uk (accessed 17 January 2008).

28. Holland B, Welch AA, Unwin ID, et al. (1991) The Composition of Foods. Cambridge: Royal Society of Chemistry and Ministry of Agriculture, Fisheries and Food.

29. Borg GA (1973) Perceived exertion: a note on "history" and methods. Med Sci Sport 5, 90-93.

30. Frayn KN (1983) Calculation of substrate oxidation rates in vivo from gaseous exchange. J Appl Physiol 55, 628-634.

31. Hardman AE \& Aldred HE (1995) Walking during the postprandial period decreases alimentary lipaemia. J Cardiovac Risk $\mathbf{2}$, $71-78$.

32. Zhang JQ, Thomas TR \& Ball SD (1998) Effect of exercise timing on postprandial lipemia and HDL cholesterol subfractions. J Appl Physiol 85, 1516-1522.

33. Weir JB (1949) New methods for calculating metabolic rate with special reference to protein metabolism. J Physiol 109, $1-9$.

34. Humphreys SM, Fisher RM \& Frayn KN (1990) Micro-method for measurement of sub-nanomole amounts of triacylglycerol. Ann Clin Biochem 27, 597-598.

35. Ullman D, Connor WE, Hatcher LF, et al. (1991) Will a highcarbohydrate, low-fat diet lower plasma lipids and lipoproteins without producing hypertriglyceridemia? Arterioscler Thromb 11, 1059-1067.

36. Antonis A \& Bersohn I (1961) The influence of diet on serum triglycerides in South African white and Bantu prisoners. Lancet 1, 3-9.

37. Ullrich IH \& Albrink MJ (1986) Physical fitness modifies carbohydrate-induced lipemia. Nutr Rep Int 33, 701-709.

38. Thompson PD, Cullinane EM, Sady SP, et al. (1988) Modest changes in high-density lipoprotein concentration and metabolism with prolonged exercise training. Circulation 78, 25-34.

39. Sidossis LS \& Mittendorfer B (1999) Effect of diet composition on triacylglycerol metabolism in humans. Clin Nutr 18, 107-109.

40. Seip RL, Mair K, Cole TG, et al. (1997) Induction of human skeletal muscle lipoprotein lipase gene expression by shortterm exercise is transient. Am J Physiol 272, E255-E261.

41. Tsetsonis NV \& Hardman AE (1996) Reduction in postprandial lipemia after walking: influence of exercise intensity. Med Sci Sport Exer 28, 1235-1242.

42. Tsetsonis NV, Hardman AE \& Mastana SS (1997) Acute effects of exercise on postprandial lipemia: a comparative study in trained and untrained middle-aged women. Am J Clin Nutr 65, 525-533.

43. Gill JM, Herd SL \& Hardman AE (2002) Moderate exercise and post-prandial metabolism: issues of dose-response. J Sports Sci 20, $961-967$. 
44. Gill JM \& Hardman AE (2000) Postprandial lipemia: effects of exercise and restriction of energy intake compared. Am J Clin Nutr 71, 465-471.

45. Merrill JR, Holly RG, Anderson RL, et al. (1989) Hyperlipemic response of young trained and untrained men after a high fat meal. Arteriosclerosis 9, 217-223.
46. Shannon KA, Shannon RM, Clore JN, et al. (2008) Aerobic exercise alters postprandial lipemia in African American versus white women. Int J Sport Nutr Exerc Metab 18, 37-48.

47. Burton FL, Malkova D, Caslake MJ, et al. (2008) Energy replacement attenuates the effects of prior moderate exercise on postprandial metabolism in overweight/obese men. Int J Obes 32, 481-489. 


\section{Appendix}
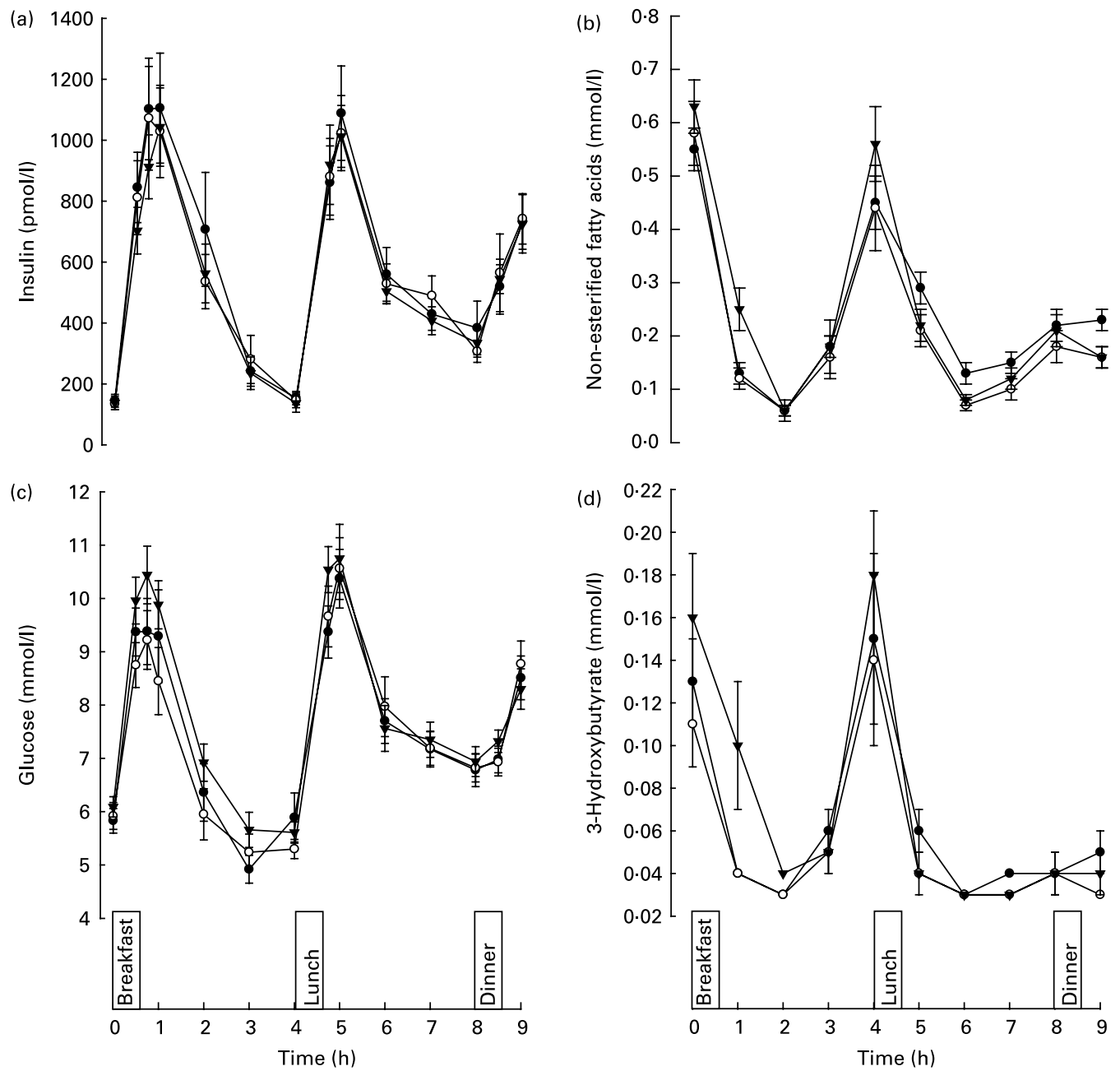

Fig. A1. (a) Plasma insulin, (b) NEFA, (c) glucose and (d) 3-hydroxybutyrate concentrations for $9 \mathrm{~h}$ while consuming (i) a typical UK diet ( $\bullet$ ), (ii) the recommended diet $(O)$ or (iii) the recommended diet with $30 \mathrm{~min}$ of brisk walking $(\mathbf{V})$. Values are means with their standard errors $(n 14)$. Insulin: main effect of time $(P<0.001)$; NEFA: main effect of time $(P<0.001)$ and trial $\times$ time interaction $(P=0.004)$; glucose: main effect of trial $(P=0.052)$ and main effect of time $(P<0.001)$; 3-hydroxybutyrate: main effect of time $(P<0.001)$. 